\title{
Twelve month follow-up on a randomised controlled trial of relaxation training for post-stroke anxiety
}

Clinical Rehabilitation I-4

(C) The Author(s) 2016

Reprints and permissions: sagepub.co.uk/journalsPermissions.nav DOI: $10.1177 / 0269215516682820$ cre.sagepub.com

@SAGE

\section{Katherine Golding ', Chris Fife-Schaw ${ }^{2}$ and Ian Kneebone ${ }^{3}$}

\begin{abstract}
Objective: To follow up participants in a randomised controlled trial of relaxation training for anxiety after stroke at 12 months.

Design: Twelve month follow-up to a randomised controlled trial, in which the control group also received treatment.

Setting: Community.

Participants: Fifteen of twenty one original participants with post-stroke anxiety participated in a one year follow-up study.

Interventions: A self-help autogenic relaxation CD listened to five times a week for one month, immediately in the intervention group and after three months in the control group.

Main measures: Hospital Anxiety and Depression Scale-Anxiety subscale and the Telephone Interview of Cognitive Status for inclusion. Hospital Anxiety and Depression Scale-Anxiety subscale for outcome. All measures were administered by phone.

Results: Anxiety ratings reduced significantly between pre and post-intervention, and between preintervention and one year follow-up $\left(\chi^{2}(2)=22.29, p<0.00 I\right)$.

Conclusions: Reductions in anxiety in stroke survivors who received a self-help autogenic relaxation $C D$ appear to be maintained after one year.
\end{abstract}

\section{Keywords}

Stroke, anxiety, self-help relaxation

Date received: 5 November 2016; accepted: I3 November 2016

'Clinical Neuropsychology Department, Great Ormond Street Hospital, UK

${ }^{2}$ School of Psychology, University of Surrey, UK

${ }^{3}$ Discipline of Clinical Psychology, Graduate School of Health,

University of Technology Sydney, Australia
Corresponding author:

Katherine Golding, Clinical Neuropsychology Department, Level 4 Frontage Building, Great Ormond Street Hospital, London WCIN 3JH, UK.

Email: katherine.golding@nhs.net 


\section{Introduction}

Mental health difficulties following stroke are common, with anxiety effecting up to a quarter of stroke survivors. ${ }^{1}$ Anxiety after stroke also persists. Only $23 \%$ of stroke survivors with 'early' anxiety (an anxiety disorder diagnosed in the first three months post-stroke) have recovered after one year. ${ }^{2}$ While anxiety is distressing post stroke it also appears to effect outcomes. It is associated with increased disability and reduced quality of life. ${ }^{3,4}$ At present evidence for effective treatment of post stroke anxiety is limited..$^{5}$ Relaxation is a promising treatment option. It appears effective for older people generally and for those with physical health complaints. ${ }^{6}$ Specific to stroke, a pilot study found self-help autogenic relaxation to be superior to a wait-list control in reducing anxiety at one, two and three months post treatment. ${ }^{7}$ It was the concern of the current study to consider whether these benefits are maintained in the longer term, that is at one year post intervention.

\section{Methods}

A detailed description of the procedure for this trial and of the original sample is available elsewhere. ${ }^{7}$ All potential participants gave written consent and were then screened, via telephone, using the Hospital Anxiety and Depression ScaleAnxiety subscale, ${ }^{8}$ cut off for inclusion $\geqslant 6^{9}$ and the Telephone Interview of Cognitive Status, cut off for inclusion $\geqslant 20 .{ }^{10}$ Initially 11 participants were randomised to receive the intervention and 10 to the waitlist control condition. The intervention group received the autogenic relaxation $\mathrm{CD}^{11}$ immediately; the control group received the $\mathrm{CD}$ after three months. All participants completed the Hospital Anxiety and Depression Scale-Anxiety subscale immediately prior to receiving the $\mathrm{CD}$ (preintervention), one month later (post-intervention) and one year after completing the intervention (one year follow-up). Figure 1 shows a flow diagram of the follow-up procedure. Participants were asked to listen to the $\mathrm{CD}$ at least five times per week for one month. After completing the intervention participants were advised that they could choose whether to continue using the $\mathrm{CD}$, and if so, how often. On all occasions the lead researcher administered the Hospital Anxiety and Depression Scale-Anxiety subscale to participants via telephone. Given the small sample size a conservative non-parametric statistical analysis approach was adopted.

\section{Results}

Fifteen $(67 \%$ male; mean age 66 years $S D=8.58)$ participated at one year follow-up (a 75\% retention rate). The mean, median, standard deviation and range of the Hospital Anxiety and Depression Scale-Anxiety subscale scores were calculated for pre and post intervention, and one year follow-up (see Table 1), along with the number of participants considered to have clinical levels of anxiety9. The Supplementary Figure visually displays the scores at screening and all follow-ups.

The anxiety ratings significantly changed between pre-intervention and subsequent followups $\left(\chi^{2}(2)=22.29, p<0.001\right)$. Wilcoxon tests were used to consider this finding in detail, with a Bonferroni correction. Median anxiety ratings significantly reduced from pre to post-intervention $(Z=-3.19, p=.001, r=0.82)$ and from pre-intervention to one year follow-up $(Z=-3.42, p=$ $.001, r=.87)$. The reduction from post-intervention to one year follow-up $(Z=-2.29, p=.022, r$ $=.75)$ did not reach significance following correction $(p<0.017)$.

\section{Discussion}

This follow up study suggests that the benefits of self-help autogenic relaxation for post-stroke anxiety persist one year later. Despite this, ten of the fifteen participants were still considered to have clinical levels of anxiety after one year suggesting that relaxation may need to be thought of as part of a stepped approach to intervention, with additional therapeutic options available to those who continue to report significant symptoms. ${ }^{12}$ It should be acknowledged that without a control group in place it is unclear whether the current 12 month followup findings reflect treatment-related change or other factors, including spontaneous remission. 


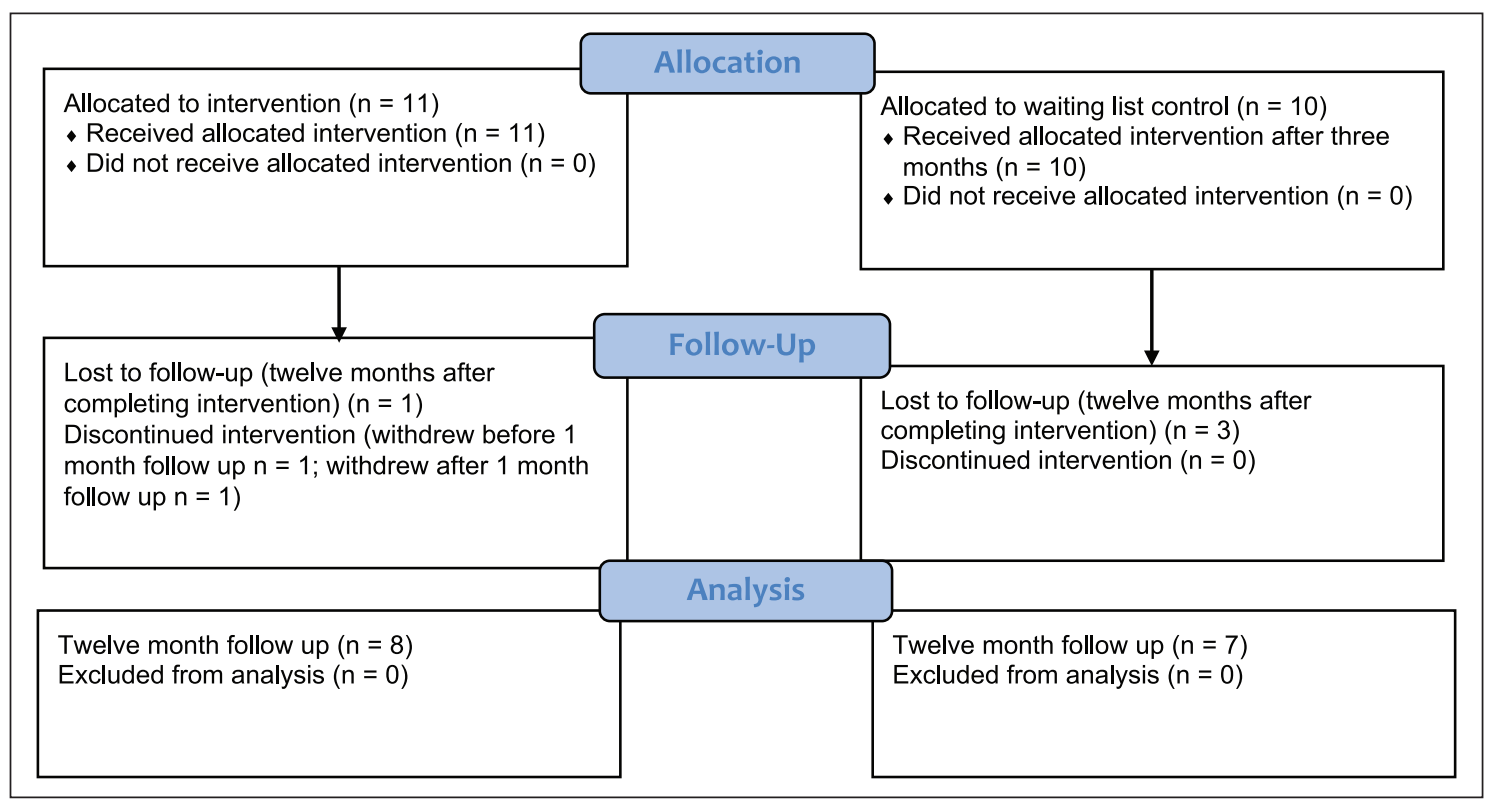

Figure I. Flow diagram showing the procedure of allocation, twelve month follow up and analysis.

Table I. Hospital Anxiety and Depression Scale - Anxiety sub-scale scores at pre-intervention, post-intervention (one month) and one year follow-up by group.

\begin{tabular}{|c|c|c|c|c|c|c|c|c|c|c|}
\hline & \multicolumn{5}{|c|}{ Intervention $(N=8)$} & \multicolumn{5}{|c|}{ Waiting list control $(N=7)$} \\
\hline & Mean & SD & Median & Range & Caseness & Mean & SD & Median & Range & Caseness \\
\hline Pre-intervention & 10.13 & 2.137 & 10 & $7-13$ & 8 & 12.57 & 3.409 & 12 & $8-19$ & 7 \\
\hline Post-intervention & 6.50 & 2.726 & 6.5 & $3-11$ & 6 & 10.00 & 2.944 & II & $6-15$ & 7 \\
\hline One year follow up & 4.63 & $\mathrm{I} .847$ & 4 & $3-8$ & 3 & 9.14 & 3.579 & 8 & $6-16$ & 7 \\
\hline
\end{tabular}

Caseness $=$ Clinical levels of anxiety, HADS-A score $\geqslant 6$.

Although the research considering the likelihood of remission of anxiety symptoms in stroke patients is limited this could be of the order of $23 \% .{ }^{2}$ Further research is needed to establish whether the benefits seen in this sample are present in a larger and more representative stroke sample including those with language and/or cognitive difficulties.

\section{Clinical message}

- The benefits of self-help relaxation in reducing post-stroke anxiety appear to persist to 12 months post treatment.

\section{Conflict of interest}

The authors declared no potential conflicts of interest with respect to the research, authorship, and/or publication of this article.

\section{Funding}

The authors received no financial support for the research, authorship, and/or publication of this article.

\section{References}

1. Campbell Burton CA, Murray J, Holmes J, et al. Frequency of anxiety after stroke: A systematic review 
and meta-analysis of observational studies. Int $J$ Stroke 2013; 8: 545-559.

2. Aström M. Generalized anxiety disorder in stroke patients a 3-year longitudinal study. Stroke 1996; 27: 270-275.

3. Ahlsiö B, Britton M, Murray $\mathrm{V}$ and Theorell $\mathrm{T}$. Disablement and quality of life after stroke. Stroke 1984; 15: 886-890.

4. Ayerbe L, Ayis A, Crichton S, et al. Natural history, predictors and associated outcomes of anxiety up to 10 years after stroke: The South London Stroke Register. Age Ageing 2014; 43: 542-547.

5. Campbell Burton CA, Holmes J, Murray J, et al. Interventions for treating anxiety after stroke. Cochrane Database of Systematic Reviews 2011; 12: CD008860.

6. Manzoni GM, Pagnini F, Castelnuovo G, et al. Relaxation training for anxiety: A ten-years systematic review with meta-analysis. BMC Psychiatry 2008; 8: 41.
7. Golding K, Kneebone I and Fife-Schaw C. Self-help relaxation for post-stroke anxiety: A randomised, controlled pilot study. Clin Rehabil 2016; 30: 174-180.

8. Zigmond A and Snaith RP. The Hospital Anxiety and Depression Scale. Acta Psychiatr Scand 1983; 67: 361-370.

9. Johnson G, Burvill PW, Anderson CS, et al. Screening instruments for depression and anxiety following stroke: Experience in the Perth community stroke study. Acta Psychiatr Scand 1995; 91: 252-257.

10. Brandt J, Spencer M and Folstein M. The telephone interview for cognitive status. Cogn Behav Neurol 1988; 1: 111-118.

11. Winkler R, James R, Fatovich B, et al. Migraine and tension headaches: A multi-modal approach to the prevention and control of headache pain. University of Western Australia: Self-Care Research Team, 1982.

12. Kneebone II. Stepped psychological care after stroke. Disabil Rehabil 2016; 38: 1836-1843. 